Original Research Paper

\title{
Concentric Circles Painting Versus Back-and-Forth Scrubbing: A Comparison Between Two Skin Disinfection Techniques
}

\author{
${ }^{1}$ Kai Beng Tan, ${ }^{1}$ Yu Cheng Chiu, ${ }^{1}$ Audrey Tham, ${ }^{1}$ Megan Hey, ${ }^{1}$ Hayley Pang, \\ ${ }^{1}$ Qian Wen Leo, ${ }^{1}$ Terilyn Ong, ${ }^{1}$ Amanda Goh and ${ }^{2}$ Sabrina Koh \\ ${ }^{1}$ Nursing Division, Sengkang General Hospital, Singapore \\ ${ }^{2}$ Nursing Education and Development, Sengkang General Hospital, Singapore
}

Article history

Received: 16-07-2021

Revised: 01-10-2021

Accepted: 05-10-2021

Corresponding Author:

Kai Beng Tan

Nursing Division, Sengkang

General Hospital, Singapore

Email: tan.kai.beng@skh.com.sg

\begin{abstract}
Traditionally, skin disinfection is performed in concentric circles. In recent years, there are studies which suggest that back-and-forth scrubbing could be a better technique. However, there is insufficient evidence to demonstrate which disinfection technique is better in reducing microbial load prior to invasive procedures. This study aims to compare the effectiveness of concentric circles painting and back-and-forth scrubbing skin disinfection techniques in reducing microorganisms' Adenosine Triphosphate (ATP) measured in Reactive Light Units (RLU). 293 participants were recruited in the quasi-experimental study. Before disinfection, the participants' bilateral antecubital fossa was swabbed using the $3 \mathrm{M}$ clean-trace surface ATP test swab (3M Clean-Trace) to detect microorganisms' ATP measured in RLU. Subsequently, on one antecubital fossa, using an assigned cleansing agent ( $0.9 \%$ normal saline or $70 \%$ isopropyl alcohol), it was disinfected by concentric circles painting. On the opposing antecubital fossa, using the same cleansing agent, it was disinfected by back-and-forth scrubbing. Thereafter, the participants' bilateral antecubital fossa was swabbed using the 3M Clean-Trace to determine the amount of ATP present post-disinfection. The pre and post disinfection median RLU and median difference in RLU between the disinfection techniques were compared. Based on 146 participants after removal of outlying data, both concentric circles painting and back-andforth scrubbing disinfection techniques significantly reduced microorganisms' ATP measured in RLU $(\mathrm{p}<0.05)$. However, between the two disinfection techniques, no significant median difference in RLU was observed ( $>0.05)$. Both concentric circles painting and back-and-forth scrubbing disinfection techniques reduced microorganisms' ATP on skin surfaces. However, no significant difference was observed between the two disinfection techniques, which could suggest that both are equally effective for skin disinfection.
\end{abstract}

Keywords: Skin Disinfection, Concentric Circle, Back-and-Forth

\section{Introduction}

Skin disinfection is one of the integral factors to prevent Hospital Acquired Infections (HAI) often caused by Catheter-Related Bloodstream Infection (CRBSI) or Surgical Site Infection (SSI) (Tsai and Caterson, 2014; Mimoz et al., 2015). During procedures that involve the breach of skin (e.g., catheter insertion, surgical incision), microorganisms from the patient's own skin flora could potentially migrate into bloodstreams or reside in surrounding tissues, resulting in undesirable CRBSI or SSI (Reichman and Greenberg, 2009; Gahloh et al., 2014). Likewise, the migration of microorganisms into bloodstreams could contaminate blood sampling, thereby affecting treatment modalities and medication choices (Hall and Lyman, 2006). Therefore, it is paramount to ensure that skin surfaces are disinfected thoroughly prior to 
invasive procedures, to prevent the detrimental effects of HAI such as poor wound healing, prolonged use of antibiotics and increased morbidity, hospitalization stay and medical cost (Hensley and Monson, 2015).

Traditionally, skin disinfection is performed by applying the cleansing agent in circular motion away from where the breach of skin occurs. This technique works well with aqueous-based cleansing agents that require a longer drying duration and it prevents contaminants from being reintroduced into the disinfected site (Baron et al., 2005). However, in recent years, the back-and-forth scrubbing technique has been suggested to be more effective in reducing microorganisms on skin surfaces. By creating friction, it facilitates the cleansing agent to penetrate deeply into multiple layers of the skin where the breach of skin occurs, thereby removing a greater amount of microorganisms in the top dermal layers of the skin (Stonecypher, 2009; Silva, 2014). Therefore, as concentric circles painting and back-and-forth scrubbing disinfection techniques are fairly divergent, this study aims to compare the effectiveness between the two techniques in reducing the amount of microorganisms' Adenosine Triphosphate (ATP) present on skin surfaces.

\section{Methods}

\section{Research Design}

The quasi-experimental study was conducted in Sengkang General Hospital (SKH), a regional hospital in Singapore from 1 September 2020 to 25 September 2020 with approval by the SingHealth Centralised Institutional Review Board, Ref: 2020/2734. The study had eight data collectors and the participants were recruited through email invitation and walk-in attendance. To be recruited into the study, the participants had to be a staff of SKH, at least 21 years old and have no allergy to $0.9 \%$ normal saline or $70 \%$ isopropyl alcohol. A total of 293 participants were recruited and written informed consents were sought. The participants were then assigned by the data collectors into two groups $(0.9 \%$ normal saline or $70 \%$ isopropyl alcohol) in an alternating manner before commencing the skin disinfection.

\section{Data Collection}

Prior to skin disinfection, the participants' bilateral antecubital fossa was swabbed using the $3 \mathrm{M}$ clean-trace surface ATP test swab (3M Clean-Trace) to detect the presence of microorganisms' ATP measured in Reactive Light Units (RLU). The swabbing was performed by the data collector in a vertical followed by horizontal plane covering an area of $5 \times 5 \mathrm{~cm}$ and it was repeated twice. The $3 \mathrm{M}$ Clean-Trace was utilised in the study as it has the capability of detecting ATP, which is an indicator of organic residues present in microorganisms and it rapidly assesses cleaning effectiveness (3M, 2020).

For participants assigned to the $0.9 \%$ normal saline group, a sterile disposable dressing set was prepared for each participant and $20 \mathrm{~mL}$ of $0.9 \%$ normal saline was poured into the dressing set. The cotton balls inside the dressing sets were soaked in $0.9 \%$ normal saline and dried briefly using the tweezers within the dressing set. For participants assigned to the 70\% isopropyl alcohol group, three pieces of individually foil wrapped $70 \%$ isopropyl alcohol swabs were prepared for each participant.

Irrespective of the participants' assigned group, all of them received both skin disinfection techniques. On one antecubital fossa, a clockwise outward concentric circles painting was performed covering an area of $5 \times 5$ $\mathrm{cm}$. The process was repeated twice and a new cotton ball was used each time. The opposing antecubital fossa was disinfected by back-and-forth scrubbing in a vertical plane moving from left to right covering an area of $5 \times 5 \mathrm{~cm}$. The process was also repeated twice.

After skin disinfection, the participants' bilateral antecubital fossa was swabbed using the 3M Clean-Trace to determine the amount of microorganisms' ATP present after disinfection. The swabbing was performed in a vertical followed by horizontal plane covering an area of $5 \times 5 \mathrm{~cm}$ and it was repeated twice.

\section{Data Analysis}

To determine the reduction of microorganisms' ATP before and after skin disinfection, the pre and post disinfection median RLU for both concentric circles painting and back-and-forth scrubbing was compared. Correspondingly, to determine the effectiveness between the disinfection techniques, the median difference in RLU of concentric circles painting and back-and-forth scrubbing was compared. Normality tests revealed that the data obtained were highly skewed and Wilcoxon Signed Rank and Mann Whitney $\mathrm{U}$ tests were performed to analyse the data. The level of significance was set at $\alpha=0.05$. All analyses were performed using the SPSS statistical software (version 14.0, SPSS, Chicago, Illinois).

\section{Results}

A total of 293 participants were recruited but only 146 were included in the analysis as uncontrollable environmental conditions (e.g., temperature, humidity) affected the microorganisms' ATP levels. Demographic profile of the participants is reflected in Table 1.

Wilcoxon Signed Rank test was performed to compare the median reduction in RLU for concentric circles painting and back-and-forth scrubbing, pre and 
post-disinfection (Table 2). As both concentric circles painting and back-and-forth scrubbing had a value of $<0.001$, there is a significant median reduction in RLU for both techniques.

To compare the efficacy of concentric circles painting and back-and-forth scrubbing technique, Mann-Whitney U test was performed. Regardless of cleansing solutions used, the median reduction between back-and-forth scrubbing and concentric circles painting technique was found to be insignificant (Table 3).
The median difference in RLU was -2956 (-6256, 1151) for concentric circles painting and $-2567(-8049,-545)$ for back-and-forth scrubbing. Since p value was 0.761 , there is no significant median reduction in RLU when comparing both techniques using $0.9 \%$ normal saline. Similarly, the median difference in RLU was -1277 (-3921, -359) for concentric circles painting and $-1826(-7892,-380)$ for $70 \%$ isopropyl alcohol, with $\mathrm{p}$ value of 0.086 , showing no significant median reduction in RLU between both techniques when using $70 \%$ isopropyl alcohol.

Table 1: Demographic profile of participants

\begin{tabular}{|c|c|c|c|c|c|}
\hline & Nursing n $(\%)$ & Medical n (\%) & Allied health n (\%) & Ancillary support n (\%) & Total n (\%) \\
\hline \multicolumn{6}{|l|}{ Gender } \\
\hline Male & $13(16.9)$ & $2(66.7)$ & $1(20.0)$ & $14(23.0)$ & $30(20.5)$ \\
\hline Female & $64(83.1)$ & $1(33.3)$ & $4(80.0)$ & $47(77.0)$ & $116(79.5)$ \\
\hline \multicolumn{6}{|l|}{ Age } \\
\hline$<30$ years old & $47(61.0)$ & $1(33.3)$ & $4(80.0)$ & $10(16.4)$ & $62(42.5)$ \\
\hline$\geq 30$ years old & $30(39.0)$ & $2(66.7)$ & $1(20.0)$ & $51(83.6)$ & $84(57.5)$ \\
\hline \multicolumn{6}{|l|}{ Race } \\
\hline Chinese & $31(40.3)$ & $2(66.7)$ & $4(80.0)$ & $27(44.3)$ & $64(43.8)$ \\
\hline Malay & $19(24.7)$ & - & - & $15(24.6)$ & $34(23.3)$ \\
\hline Indian & $8(10.3)$ & - & - & $12(19.7)$ & $20(13.7)$ \\
\hline Others & $19(24.7)$ & $1(33.3)$ & $1(20.0)$ & $7(11.4)$ & $28(19.2)$ \\
\hline \multicolumn{6}{|l|}{ Nationality } \\
\hline Singaporean & $44(57.1)$ & $2(66.7)$ & $3(60.0)$ & $36(59.0)$ & $85(58.2)$ \\
\hline Others & $33(42.9)$ & $1(33.3)$ & $2(40.0)$ & $25(41.0)$ & $61(41.8)$ \\
\hline
\end{tabular}

Table 2: Median Relative Light Units (RLU) based on skin disinfection technique

\begin{tabular}{|c|c|c|}
\hline \multicolumn{3}{|c|}{$0.9 \%$ normal saline $(n=83)$} \\
\hline & Concentric circles painting RLU & Back-and-forth scrubbing RLU \\
\hline & Median (IQR) & Median (IQR) \\
\hline Pre-disinfection & $4572(2100,10959)$ & $4428(1508,14383)$ \\
\hline Post-disinfection & $1504(387,4442)$ & $1300(336,4243)$ \\
\hline $\mathrm{p}$ value & $<0.001$ & $<0.001$ \\
\hline \multicolumn{3}{|c|}{$70 \%$ isopropyl alcohol $(n=63)$} \\
\hline & Concentric circles RLU & Back-and-forth scrubbing RLU \\
\hline & Median (IQR) & Median (IQR) \\
\hline Pre-disinfection & $3521(1084,8157)$ & $3115(819,14299)$ \\
\hline Post-disinfection & $1630(475,3181)$ & $1328(259,4047)$ \\
\hline $\mathrm{p}$ value & $<0.001$ & $<0.001$ \\
\hline
\end{tabular}

Table 3: Median difference Relative Light Units (RLU) based on skin disinfection technique

\begin{tabular}{lll}
\hline & $0.9 \%$ normal saline $(\mathrm{n}=83)$ & $70 \%$ isopropyl alcohol $(\mathrm{n}=63)$ \\
\hline Concentric circles RLU & & $-1277(-3921,-359)$ \\
Median (IQR) & $-2956(-6256,-1151)$ & $-1826(-7892,-380)$ \\
Back-and-forth scrubbing RLU & $-2567(-8049,-545)$ & 0.086 \\
Median (IQR) & 0.761 & value
\end{tabular}

\section{Discussion}

Based on this study, the authors found that both disinfection techniques showed a significant reduction in microorganisms' ATP regardless of the cleansing solution used. Furthermore, as the comparison of median difference in RLU between concentric circles painting and back-and-forth scrubbing was not significant, this could suggest that both disinfection techniques are equally effective for skin disinfection.

The findings from this study were similar to Lundberg et al. (2016) which compared two disinfection techniques. On one ankle, the surgical site was initially disinfected using $2 \%$ chlorhexidine gluconate with $70 \%$ isopropyl by repeated back-and-forth stroke. On the opposite ankle, using povidone iodine, the site was 
initially scrubbed followed by centric circles application. Both disinfection techniques showed a reduction in Colony Forming Units (CFU) after the skin disinfection, but no significant difference was observed between the two disinfection techniques.

Likewise, Vagholkar and Julka (2012) studied surgical skin preparation involving iodophore painting in comparison to iodophore scrubbing followed by painting. While the study did not measure the reduction of microorganisms after skin disinfection, it was found that four patients developed SSI. However, no significant association between the disinfection techniques and development of SSI could be ascertained.

Thus, based on the studies performed by Lundberg et al. (2016) and Vagholkar and Julka (2012), it could imply that an additional attempt to scrub or paint the intended site before or after disinfection may not be beneficial in reducing the amount microorganisms or preventing SSI. Correspondingly, as demonstrated in this study, performing either concentric circles painting or backand-forth scrubbing is equally effective in reducing microorganisms' ATP on skin surfaces.

Nonetheless, it was observed that these previous studies differ in disinfection techniques, cleansing agents and outcome measurements. Whereas, in this study, to identify the most effective disinfection technique, each participants received only one cleansing solution $(0.9 \%$ normal saline or $70 \%$ isopropyl alcohol) for both concentric circles painting and back-and-forth scrubbing techniques.

The authors acknowledge that to determine the ideal disinfection technique, the use of agar plates to culture CFU, monitoring postoperative SSI, or observing blood culture contamination rates would be preferred. While the $3 \mathrm{M}$ Clean-Trace is not a conventional tool to detect the presence of microorganisms on skin surfaces, it was selected based on its suitability as there was an ongoing pandemic during the time of study; Coronavirus Disease 2019 (COVID-19). The pandemic caused all available laboratory resources to be prioritised for COVID-19 testing and using agar plates to culture CFU was not possible due to lean manpower and resources. Unfortunately, the novelty of using the $3 \mathrm{M}$ Clean-Trace resulted in substantial data being voided due to oversensitivity of the device towards environmental conditions.

\section{Conclusion}

Both concentric circles painting and back-and-forth scrubbing demonstrated a decrease in microorganisms' ATP on skin surfaces after disinfection. Despite so, comparison between the two disinfection techniques showed no significant difference. Henceforth, both disinfection techniques are equally effective and it is ideal to consider the patient's skin condition before selecting the appropriate skin disinfection technique.

\section{Acknowledgement}

The authors would like to acknowledge Hui Cheng Tan from Clinical Governance, Sengkang General Hospital for performing the statistical analyses of this study.

\section{Declaration}

The authors declare that there is no conflict of interest and this study was not supported by any grants from public agencies, commercial or not-for-profit sectors.

\section{Author's Contributions}

All authors equally contributed in this work.

\section{Ethics}

This study was approved by the SingHealth Centralised Institutional Review Board, Ref: 2020/2734 and all participants had consented to the study.

\section{References}

3M. (2020). 3M Clean-Trace Surface ATP Test Swab. Baron, E. J., Weinstein, M. P., Dunne, W. M., Yagupsky, P., Welch, D. F., \& Wilson, D. M. (2005). Cumitech $1 \mathrm{C}$, blood cultures IV. Baron EJ, co-ordinating editor. ASM Press, Washington, DC.

Gahloh, R., Nigam, C., Kumar, V., Yadav, G., \& Anupurba, S. (2014). International Journal of Critical Illness and Injury Science, 4(2), 162-167, doi.org/10.4103/2229-5151.134184

Hall, K. K., \& Lyman, J. A. (2006). Updated review of blood culture contamination. Clinical Microbiology Reviews, 19(4), 788-802. doi.org/10.1128/CMR.00062-05

Hensley, B. J., \& Monson, J. R. (2015). Hospital-acquired infections. Surgery (Oxford), 33(11), 528-533. doi.org/10.1016/j.mpsur.2015.08.008

Lundberg, P. W., Smith, A. A., Heaney, J. B., Wimley, W. C., Hauch, A. T., Nichols, R. L., \& Korndorffer Jr, J. R. (2016). Pre-operative antisepsis protocol compliance and the effect on bacterial load reduction. Surgical Infections, 17(1), 32-37. doi.org/10.1089/sur.2015.107

Mimoz, O., Lucet, J. C., Kerforne, T., Pascal, J., Souweine, B., Goudet, V., ... \& CLEAN Trial Investigators. (2015). Skin antisepsis with chlorhexidine-alcohol versus povidone iodine-alcohol, with and without skin scrubbing, for prevention of intravascular-catheterrelated infection (CLEAN): An open-label, multicentre, randomised, controlled, two-by-two factorial trial. The Lancet, 386(10008), 2069-2077. doi.org/10.1016/S0140-6736(15)00244-5

Reichman, D. E., \& Greenberg, J. A. (2009). Reducing surgical site infections: A review. Reviews in Obstetrics and Gynecology, 2(4), 212.

https://www.ncbi.nlm.nih.gov/pmc/articles/pmc28 12878/ 
Kai Beng Tan et al. / International Journal of Research in Nursing 2021, Volume 12: 21.25 DOI: 10.3844/ijrnsp.2021.21.25

Silva, P. (2014). The right skin preparation technique: A literature review. Journal of perioperative practice, 24(12), 283-285. doi.org/10.1177/175045891402401204

Stonecypher, K. (2009). Going around in circles: is this the best practice for preparing the skin?. Critical Care Nursing Quarterly, 32(2), 94-98. doi.org/10.1097/CNQ.0b013e3181a27b86
Tsai, D. M., \& Caterson, E. J. (2014). Current preventive measures for health-care associated surgical site infections: A review. Patient Safety in Surgery, 8(1), 1-13. doi.org/10.1186/s13037-014-0042-5

Vagholkar, K., \& Julka, K. (2012). Preoperative skin preparation: Which is the best method. The Internet Journal of Surgery, 28(4). 